Reflux

\section{Gatekeeper reflux repair system; a mechanistic hypothesis}

\section{P J Kahrilas, T J Lee}

The Gatekeeper reflux repair system is the fifth endoluminal therapy for GORD to gain regulatory approval and, akin to its predecessors, still faces many questions regarding its place in clinical practice

$\mathrm{A}^{\mathrm{s}}$ lthough there can clearly be an argument regarding the threshold at which it becomes a disease, gastro-oesophageal reflux disease (GORD) is ultimately the result of excessive gastro-oesophageal reflux and the associated consequences of that. It then seems rather straightforward that therapeutic interventions should seek to reduce or eliminate gastrooesophageal reflux. The paradox is that the dominant medical interventions do not; rather, they alter the content of the refluxate (by inhibiting gastric acid secretion) so as to make it less noxious to the oesophageal epithelium. This, in a nutshell, summarises the decades old argument regarding the relative merits of medical verses surgical therapy for GORD. Or so it was, until the dawn of the era of endoluminal therapies for GORD a few short years ago. Now it seems that physicians have assumed some attributes of their surgical colleagues as they experiment with therapies that target the reflux itself.

The Gatekeeper reflux repair system is the fifth endoluminal therapy for GORD to gain regulatory approval either in the European Union or in the USA (Stretta, Endocinch, Enteryx, and NDO Plicator). As detailed by Cicala and colleagues ${ }^{1}$ in this issue of Gut (see page 183) and by Fockens et al in a recent summary report of a multicentre study, ${ }^{2}$ the concept behind Gatekeeper is to restrict the luminal dimensions of the distal oesophagus by the submucosal implantation of a relatively bioinert hydrogel material. The intended consequence of this is not necessarily to alter lower oesophageal sphincter (LOS) pressure, length, or relaxation characteristics, but to restrict the aperture through which gastrooesophageal refluxate must flow. Flow through a tube, be it round or elliptical, is highly dependent on both the cross sectional area of the tube and the viscosity of the fluid flowing through it. $^{34}$ Thus to use the example of transient LOS relaxation, the effect of the Gatekeeper procedure would not be to alter the frequency with which these occur but to change the consequences of their occurrence. Whereas prior to implantation the opening dimensions during transient LOS relaxations allow for voluminous reflux of gas or gastric juice, afterwards reflux is relatively restricted to gas (owing to its viscosity being 55 -fold less than water). In essence, the effect of the Gatekeeper implantation is to reverse the oesophagogastric junction opening anomalies recently described in GORD patients compared with normals. ${ }^{4}$

So, are the data presented herein consistent with the above mechanistic hypothesis? Cicala and colleagues ${ }^{1}$ investigated the effect of the Gatekeeper treatment in nine patients on $\mathrm{pH}$ data, oesophageal manometry, and GORD-health related quality of life scores. $\mathrm{pH}$ data were obtained at $5 \mathrm{~cm}$ above the manometrically identified LOS and both 3 and $10 \mathrm{~cm}$ below the upper oesophageal sphincter prior to treatment and six months afterwards. They found that the distal acid exposure time was unchanged (quite possibly due to a type 2 error given the small number of subjects studied). However, reflux detected by both more proximally positioned sensors decreased substantially. Conceptually, reflux was still occurring but at a slower flow rate and, hence, with less proximal migration. Given that existing physiological data support the notion that an important determinant of reflux symptom generation is the proximal extent of mucosal acidification, ${ }^{56}$ this observation offers a mechanistic explanation for the observed therapeutic effect of Gatekeeper. Similarly, in the Fockens study ${ }^{2}$ of 68 patients (49 of whom were followed for six months), the treatment resulted in less oesophageal acid exposure and less regurgitation. In both studies, these effects were associated with substantial symptom reduction, albeit with an uncontrolled study design. So, yes, these data do argue for a relevant therapeutic effect.
Given the above data, does this now mean that the Gatekeeper treatment is ready for widespread application? Clearly, not yet. There are many aspects to a treatment modality that define its optimal utilisation. With respect to an endoluminal therapy for GORD, one must consider: (1) safety, (2) efficacy, (3) cost, (4) durability, and (5) reversibility. In terms of safety and reversibility, the data on Gatekeeper are encouraging. The few complications reported in the multicentre study were easily remedied by removing the implants. ${ }^{2}$ Cost has yet to be determined but, as with other endoscopic therapies, will likely be somewhere between that of chronic proton pump inhibitor use and surgical fundoplication. Thus the dominant unresolved questions are of efficacy and durability, and much remains to be learned in these domains. Fockens et al reported that $70.4 \%$ of prostheses were retained at six months. ${ }^{2}$ These authors also found a statistical correlation between the number of retained prostheses and the quality of the clinical response. However, major issues remain to be addressed. Who are the ideal patients to treat? Is oesophagitis or hiatus hernia a relative contraindication? Where is the optimal implant location? What is the optimal number of implants per treatment? Is this number the same for every patient or is it dependent on other variables? How long will the implants remain in place? Is retreatment feasible? How much of the observed treatment effect is a placebo response? These and a myriad of other questions can only be answered by further studies and it is imperative that such studies utilise appropriate controls in their design. Encouragingly, one such study has recently been initiated. Hopefully, that ongoing, multicentred, randomised, sham controlled study of GORD patients with mild or absent oesophagitis will shed light on the most fundamental question of all: the magnitude of the Gatekeeper treatment effect.

\section{ACKNOWLEDGEMENT}

This work was supported by grant ROI DC00646 (PJK) from the Public Health Service

Gut 2005;54:179-180.

doi: $10.1136 /$ gut.2004.051805

\section{Authors' affiliations}

P J Kahrilas, T J Lee, Department of Medicine, Northwestern University's Feinberg School of Medicine, Chicago, Illinois 60611, USA

Correspondence to: Dr P J Kahrilas, Northwestern University, Feinberg Śchool of Medicine, Division of Gastroenterology, Department of Medicine, 676 N St Clair Street, 
Suite 1400, Chicago, Illinois 60611, USA; p-kahrilas@northwestern.edu

Conflict of interest: None declared.

\section{REFERENCES}

1 Cicala M, Gabbrielli A, Emerenziani S, et al. Effect of endoscopic augmentation of the lower oesophageal sphincter (Gatekeeper reflux repair system) on intraoesophageal dynamic characteristics of acid reflux. Gut 2005;54:183-6.
2 Fockens $\mathbf{P}$, Bruno MJ, Gabbrielli A, et al. Endoscopic augmentation of the lower oesophageal sphincter for the treatment of gastrooesophageal reflux disease: multicenter study of the gatekeeper reflux repair system. Endoscopy 2004;36:682-9.

3 Pandolfino JE, Shi G, Curry J, et al. Esophagogastric junction distensibility: a factor contributing to sphincter incompetence. Am J Physiol Gastrointest Liver Physiol 2002;282: G1052-8.

4 Pandolfino JE, Shi G, Trueworthy B, et al. Esophagogastric junction opening during relaxation distinguishes non-hernia reflux patients, hernia patients and normals. Gastroenterology 2003; 125:1018-24.

5 Weusten BL, Akkermans $L M$, vanBergeHenegouwen GP, et al. Symptom perception in gastroesophageal reflux disease is dependent on spatiotemporal reflux characteristics. Gastroenterology 1995; 108:1739-44.

6 Cicala M, Emerenziani S, Caviglia R, et al. Intraoesophageal distribution and perception of acid reflux in patients with non-erosive gastrooesophageal reflux disease. Aliment Pharmacol Ther 2003;18:605-13.

\section{Granuloma formation in the different phenotypes of Crohn's disease}

\section{F Hofstaedter}

\section{Granulomas may be more than diagnostic tools and could be significant in the biology and clinical course of Crohn's disease}

O ur immunological and molecular knowledge base $^{1}$ in idiopathic chronic inflammatory bowel disease (IBD) has seen a tremendous increase over the past few years. Laboratory application of sophisticated new methodologies has revealed a plethora of agonistic and antagonistic factors involved in the pathogenesis of both ulcerative colitis and Crohn's disease. Proinflammatory and Th2 derived anti-inflammatory cytokines are secreted by and act on various different cell types by forming a complex network of interdigitating molecular pathways with profound effects on epithelial cells, lymphocytes, endothelial cells, and monocytes. This multilayered interplay of humoral factors and various cells at different stages of differentiation appears similar to a symphony where the end result is perfect yet the role of various single instruments remains obscure to the casual listener.

Some of the newly discovered pathways have been utilised to develop new therapeutic strategies ${ }^{2}$ and molecular genetic studies in particular have provided fascinating new insights into the problems of genetic host susceptibility. Polymorphisms or mutations in the NOD2 gene $^{34}$ are coding for proteins involved in the recognition of different pathogen associated molecular patterns and different bacteria, ultimately resulting in the activation of nuclear factor $\kappa \mathrm{B}$. Other members of the innate immune system such as Toll-like receptors appear to be involved in Crohn's disease. 56
Histopathological features of IBD are well defined and little if any changes have occurred during the past few years. Any modern textbook on the subject of IBD will certainly contain a single chapter on histopathology but only the diagnostic role of histopathology in IBD is emphasised. While this remains especially true for the differential diagnosis of Crohn's disease versus ulcerative colitis, the problem of dysplasia, and issues of inter- and intraobserver variability, attempts to correlate morphology with molecular and immunological mechanisms are rarely found. Granulomas for example are seen as diagnostic tools but their potential role in disease biology is neglected. This is contrasted by numerous basic research publications on IBD in which the authors do not mention granulomas at all and where it remains doubtful whether they have ever seen such lesions.

There have been only a few recent reports on the frequency of granulomas in endoscopic and surgical specimens of patients with Crohn's disease and prospective population based studies are lacking. The article of Heresbach and colleagues $^{7}$ in this issue of Gut carefully defines the histomorphological features of epithelioid cell granulomas, microgranulomas, and isolated giant cells (see page 215). Correlating the morphological findings with treatment data and clinical outcome revealed that epithelioid cell granulomas might indicate a more aggressive clinical course. The article by Pierik and colleagues, ${ }^{8}$ also in this issue of Gut, compares the occurrence of epithelioid cell granulomas with some of the most important genetic variants of the innate immune system (CARD15/NOD2 and Toll-like receptor 4) in a well defined cohort of patients with Crohn's disease (see page 223). The lack of significant correlations between the prevalence of epithelioid cell granulomas and immune system variants is disappointing but other findings, such as a higher frequency of granulomas in distal portions of the intestine and in younger patients, are of interest.

Sampling error may have influenced the detection rate of epithelioid cell granulomas in both studies but the morphological and molecular data are well presented and prospectively correlated. This is especially important as most of the basic science in IBD research generates functional data from in vitro experiments or laboratory animal models.

Epithelioid granulomas seen in Crohn's disease show a specific geographic arrangement with multinuclear giant cells and T lymphocytes, similar to granulomas observed in many infectious diseases. Specific disease phenotypes may provide insights towards answering the question of whether a phenomenon is causative in Crohn's disease or ulcerative colitis, or simply reflects secondary inflammatory reactions.

Both articles contribute to this question by stressing the significance of epithelioid cell granulomas in the biology and clinical course of the disease. Tremendous progress in the understanding of complex immunological networks and genetic susceptibility provides a new stimulus to re-evaluate certain morphological features of disease. Granulomas may in fact contain previously unsuspected biological information that goes beyond their established role as a diagnostic tool.

Gut 2005;54:180-181.

doi: 10.1136/gut.2004.048082

Correspondence to: Dr F Hofstaedter, Institute of Pathology, University of Regensburg, F J Strauss Allee 11, Regensburg D 93042, Germany; ferdinand.hofstaedter@

klinik.uni-regensburg.de 


\section{REFERENCES}

1 Taylor KD, Rotter Jl, Yang H. Genetics of inflammatory bowel disease. In: Targan SR, Shanahan F, Karp LC, eds. Inflammatory bowel disease:from bench to bedside, 2nd edn Dordrecht: Kluwer Academic, 2003:21-65.

2 Targan SR, Hanauer SB, Vandeventer SJH, et al. A short term study of chimeric monoclonal antibody $\mathrm{Ca} 2$ to tumor necrosis factor alpha for Crohn's disease. NEngl J Med 1997;337: 1029-35.

3 Hugot JP, Chamaillard M, Zouali $\mathrm{H}$, et al. Association of NOD2 leucine-rich repeat variants with susceptibility to Crohn's disease. Nature 2001;411:599-603

4 Ogura $\mathrm{Y}$, Bonen DK, Inohara $\mathrm{N}$, et al. A frame shift mutation in NOD2 associated with susceptibility to Crohn's disease. Nature 2001;411:603-6.

5 Cario E, Podolsky DK. Differential alteration in intestinal epithelial cell expressions of Toll-like receptor 3 (TLR3) and TLR4 in inflammatory bowel disease. Infect Immun 2000;68:7010-17.

6 Franchimont D, Vermeire S, El Housni $\mathrm{H}$ et al. Deficient host bacteria interactions

\author{
in inflammatory bowel disease? The \\ Toll-like receptor (TLR4)-A sp299Gly \\ polymorphism is associated with Crohn's \\ disease and ulcerative colitis. Gut \\ 2004;53:987-92. \\ 7 Heresbach D, Alexandre JL, Branger B, et al. \\ Frequency and significance of granulomas in a \\ cohort of incident cases of Crohn's disease. Gut \\ 2005:54:215-22. \\ 8 Pierik M, De Hertogh G, Vermeire S, et al. \\ Epithelioid granulomas, pattern recognition \\ receptors, and phenotypes of Crohn's disease. \\ Gut 2005;54:223-7.
}

\section{Value of MR colonography for assessment of inflammatory bowel disease? Believe what you see-see what you believe}

\section{Gasche, K Turetschek}

\section{Should magnetic resonance colonography be used to assess colonic inflammation in known inflammatory bowel disease or for assessment of inflammatory bowel disease?}

\begin{abstract}
"libenter homines id quod volunt credunt." (Men willingly believe what they wish). Gaius Julius Caesar, De Bello Gallico, Book 3.
\end{abstract}

I maging methods in inflammatory bowel disease (IBD) are used to serve two purposes: firstly, to establish the diagnosis in suspected IBD, and secondly, to gain information for correct management in known cases of IBD. To date, colonoscopy with biopsy remains the method of first choice to diagnose IBD. Discrete morphological alterations such as erythema, oedema, and granularity of the mucosa, small erosions, or aphthous ulcers can be reliably depicted by videoendoscopy and subsequently confirmed by histopathology. In established IBD however, patients and physicians are reluctant to perform repeated colonoscopies because of the invasive nature of the test and the inability to assess extraluminal complications in Crohn's disease, including enteric fistulae or abscesses. For this purpose, cross sectional imaging methods have gained increasing importance in the past years. ${ }^{1}$ The question arises as to whether advancement in technology (multislice computed tomography or magnetic resonance imaging (MRI) scans) may also enable assessment of the mucosal inflammation in IBD, thereby potentially replacing endoscopy and biopsy at some point in the future.

This question was approached by two independent groups from Germany in this issue of Gut. $^{23}$ The group from Essen $^{2}$ reported on a two phase investigation (see page 257). ${ }^{2}$ Firstly, they established the method of MR colonography (an MRI examination with large bowel enema for luminal distension) in healthy subjects and precisely scored several imaging parameters, such as bowel wall thickening, contrast media enhancement of the bowel wall, number of lymph nodes or haustral folds, as well as imaging artefacts. Next they tested its diagnostic accuracy in patients with known IBD. Sixty eight of 73 segments were identified as inflamed (by applying a specific MRI based score and comparing it with the results of histopathology as reference); there were no false positives, resulting in sensitivity and specificity values of $87 \%$ and $100 \%$, respectively. The authors concluded that MR colonography is a promising alternative to endoscopy in monitoring IBD patients.

The second paper in this issue of $G u t^{3}$ reached a somehow different conclusion. Applying an authentic prospective design, the group from Regensburg compared MRI colonography with conventional endoscopy using bowel wall thickening and contrast enhancement as MRI determinants of inflammation (see page 250). From 154 total bowel segments investigated, the authors achieved sensitivity and specificity values of $58.8 \%$ and $91.4 \%$ in ulcerative colitis, and $31.6 \%$ and $100 \%$ in Crohn's disease, respectively. The authors concluded that MR colonography is not suitable to adequately assess colonic inflammation in patients with IBD, with the exception of severe Crohn's disease.

It is likely that these contrary conclusions may confuse gastroenterologists as well as radiologists. Can we use MR colonography to adequately assess colonic inflammation in known IBD or not? Do radiologists believe what they see and or do they see what they believe? Can gastroenterologists still trust radiology reports?

Both studies were done in the same geographic region, in a comparable university hospital based setting, using a similar study design. Delving into the details of both studies, however, a series of small differences arises that may have contributed to the optimistic conclusion in Essen and at the same time to the pessimistic view in Regensburg.

The group in Essen ${ }^{2}$ took advantage of the latest technological MRI equipment. They used a stronger gradient system (compared with the Regensburg group) in conjunction with the latest developments in the software sector (3D-VIBE sequence). By testing the feasibility of their technique in healthy subjects, they established sensitive measures for correctly defining normal colonic segments. Both issues may have improved discriminating normal from diseased bowel.

However, the superior results from the Essen radiologists ${ }^{2}$ may have been caused by a certain amount of selection bias in their study. Firstly, the investigators limited their trial to highly active (applying both clinical and serological markers of active disease) individuals with known large bowel inflammation. Hence they studied only sick and symptomatic cases with a high probability of significant bowel changes. Secondly, they excluded any colonoscopic normal segments from their comparative 
analysis by not taking biopsies from normal appearing tissue (although this is recommended in their national guidelines). ${ }^{45}$ Thereby, their analyses were limited to endoscopically and histologically inflamed segments in a series of active colitis patients. Lastly, the report is unclear on the method of histological grading, on the rational and procedures for developing the radiological score of inflammation, and on the numerical results in the IBD population.

The histological features of Crohn's disease differ from ulcerative colitis. Therefore, a single histological grading system as a gold standard of inflammation is questionable. The mismatch between endoscopic disease activity and histopathology has been known for several years. Specifically in Crohn's disease, physicians are facing a multidimensional dilemma: clinical activity correlates poorly with endoscopic activity or histopathology. In fact, most endoscopic studies failed to demonstrate a relationship between disease activity, disease severity, endoscopic findings, or the degree of inflammation in biopsies (reviewed by Geboes and Dalle ${ }^{6}$ ). With regard to the novel MRI based score for quantification of bowel inflammation, two independent groups of IBD patients should have been studied: one for establishing and another for validating this score.

The Regensburg group ${ }^{3}$ avoided such selection bias by enrolling consecutive IBD patients, regardless of IBD subtype, Crohn's disease location (including noncolonic Crohn's disease), or disease activity. Thereby, they appropriately mimicked the true clinical situation and tested MR colonoscopy rigorously against conventional colonoscopy. However, they could have improved their sensitivity in two ways: firstly, by studying the normal cut off values in a healthy population, and secondly, by using quantitative measures for the contrast to noise ratio and contrast enhancement.

When critically interpreting the findings from Essen, ${ }^{2}$ MR colonography was able to reliably depict inflamed bowel segments in patients with known and systemically active colitis, a conclusion that is quite similar to that of the Regensburg group. ${ }^{3}$ In addition, MR colonography is certainly useful in identifying extraluminal disease complications, such as fistulae, enlarged mesenteric lymph nodes, or abscesses, with the advantage of a non-invasive and radiation free examination.

Will MR colonography replace colonoscopy in the future? We do not believe so. However, it is human nature that men willingly believe what they wish. So too does the group from Essen, by blaming colonoscopy and biopsy as sources of bowel perforation in IBD. ${ }^{2}$ The fact is that bowel perforation is a rare complication that occurs in approximately $0.045 \%$ of patients undergoing diagnostic colonoscopy ${ }^{7}$ and is not related to biopsy of inflamed and thickening bowel walls. On the other hand, the technique and spatial resolution of MR colonography will be further advanced in the future and thus successfully used in IBD patients. Instead of replacing colonoscopy in the future however, we believe that MR colonography will be complementary, similar to the situation with MR cholangiography and ERCP.

Gut 2005;54:181-182.

doi: $10.1136 /$ gut. 2004.044982

\section{Authors' affiliations}

C Gasche, Department of Medicine, Medical

University Vienna, Austria

K Turetschek, Department of Radiology,

Medical University Vienna, Austria

Correspondence to: Professor C Gasche Medical University Vienna, Neues AKH Klinik Innere Medizin 4, Vienna A-1090, Austria; christoph.gasche@meduniwien.ac.at

Conflict of interest: None declared.

\section{REFERENCES}

1 Turetschek K, Gasche C. Current status and new imaging trends in IBD. In: Bernstein $\mathrm{CN}$, ed. Inflammatory Bowel Disease Yearbook. London: Remedica Publishing Ltd, 2003.

2 Ajaj WM, Lauenstein TC, Pelster G, et al. Magnetic resonance colonography for the detection of inflammatory diseases of the large bowel: quantifying the inflammatory activity. Gut 2005;54:257-63.

3 Schreyer AG, Rath HC, Kikinis R, et al. Comparison of magnetic resonance imaging colonography with conventional colonoscopy for the assessment of intestinal inflammation in patients with inflammatory bowel disease: a feasibility study. Gut 2005;54:250-6.

4 Riemann JF. Guidelines of the DGVS. Clinical diagnosis. German Society of Digestive and Metabolic Diseases. Z Gastroenterol 2001;39:21-4.

5 von Herbay A. Guidelines of the DGVS. Histopathological diagnosis. German Society of Digestive and Metabolic Diseases. Z Gastroenterol 2001;39:25-7.

6 Geboes K, Dalle I. Influence of treatment on morphological features of mucosal inflammation. Gut 2002;50(suppl 3):11137-42.

7 Waye JD, Kahn O, Auerbach ME. Complications of colonoscopy and flexible sigmoidoscopy. Gastrointest Endosc Clin N Am 1996;6:343-77. 